\title{
CONDICÕES DE SAÚDE BUCAL DE ESCOLARES DE ZONA RURAL DO RIO GRANDE DO SUL
}

\section{THE ORAL HEALTH CONDITIONS OF SCHOOLCHILDREN FROM A RURAL AREA OF RIO GRANDE DO SUL}

\author{
Márcia Cançado Figueiredo* \\ Vinícius Mendes Felten** \\ Cássia Mendes da Silva*** \\ Kethlen Pinzon de Oliveria**** \\ Kátia Valenca Correia Leandro da Silva*****
}

\begin{abstract}
Resumo: Às áreas rurais brasileiras, apresentam os piores indicadores de renda, saneamento básico e de níveis de escolaridade quando comparadas às localizações urbanas e, este fato pode configurar um importante agravo à saúde geral e bucal de seus moradores. Deste modo, diante deste contexto o objetivo do presente trabalho foi descrever os resultados de uma atividade de extensão interdisciplinar em saúde que avaliou as condições de saúde bucal de estudantes de idade entre 5 a 15 anos, residentes em uma zona rural do estado do Rio Grande do Sul e avaliar a concentração de íons de fluoreto nas águas de abastecimento naturais da região. Durante um mutirão de saúde de uma extensão universitária, foram realizadas avaliações odontológicas, preenchimento do questionário de nível sócio econômico dos cuidadores dos escolares e coleta da água em 3 poços artesianos da região.. Constatou-se que aproximadamente $50 \%$ dos escolares apresentaram lesões cariosas, placa dentária, sangramento gengival e um grau severo de fluorose. Eles estavam desprovidos de assistência odontológica, preventiva, curativa e viviam sem saneamento básico adequado. Os teores de íons de fluoreto nas águas de abastecimento natural não se encontravam dentro dos padrões de normalidade.
\end{abstract}

Palavras chave: Assistência à saúde; escolares; cárie dentária; fluorose dentária.

\begin{abstract}
This study demonstrates that Brazilian rural areas present the worst indicators in income, basic sanitation and schooling, if it is compared to urban areas, which may represent a major problem to their oral and general health. Thus, this study aimed to describe the results of a health interdisciplinary outreach activity that evaluated the oral health conditions of students, aged 5 to 15, from a rural area of Rio Grande do Sul, Brazil, and to measure the fluoride of the water consumed by them. The data were collected through participants' dental evaluation, social economic questionnaire and analisis of waters collected from three artesian wells. It was found that approximately $50 \%$ of the students had carious lesions, dental plaque, gingival bleeding and some degree of mild to severe fluorosis. It was noticed that the comunity were devoided of dental care, preventive and curative assistance, and were without adequate basic sanitation facilities. Besides, the levels of fluoride ions in the natural sources of waters fell below the standards
\end{abstract}

Keywords: Health care; students; dental caries; dental fluorosis. 


\section{Introdução}

Existe uma associação entre desenvolvimento social e cárie dentária, tanto no campo do indivíduo quanto no coletivo, sendo que a prevalência de cárie dentária é maior para populações de baixo nível socioeconômico e cultural (BALDANI; VASCONCELOS; FERREIRA, 2004). A baixa renda pode estar associada ao grau de educação, valor atribuído à saúde, estilo de vida, acesso à informação sobre cuidados de saúde e, como consequência, pode ser um fator indireto para a susceptibilidade à cárie dentária. (SPOLIDORIO, 2003).

De acordo com Gambhir et al. (2013), as doenças bucais estão entre as mais comuns e prevalentes em todo o mundo, sendo que uma condição de saúde bucal precária pode ter um impacto significativo na qualidade de vida das crianças e adolescentes, podendo levar à deterioração da sua saúde em geral. Para Campos et al., em 2010, a cárie dentária e a doença periodontal estão associadas a outras condições além daquelas relacionadas ao meio bucal de uma pessoa.

Com relação às crianças de 12 anos de idade, o último levantamento epidemiológico realizado em 2010 no Brasil (SBBrasil, 2010) demonstrou que as alterações bucais estão presentes: $56,50 \%$ - cárie dentária; $34 \%$ - sangramento gengival e cálculo dentário; $20,5 \%$ - traumatismos; $38,80 \%$ - oclusopatias e $16,7 \%$ - fluorose. Da mesma forma, foram realizadas estimativas para a condição socioeconômica, sendo avaliada pela renda familiar e a escolaridade.

A partir dessa análise, expressa-se a desigualdade regional e social: os estados do Norte e Nordeste do Brasil apresentam piores condições socioeconômicas e de escolaridade se comparados aos estados do Sul e Sudeste (BRASIL, 2011). Cabe ainda aqui destacar que, no estado do Rio Grande do Sul, 4.125.995 pessoas (2.161.121 de homens e 1.964.874 de mulheres) vivem na zona rural, caracterizada como uma "região deprimida", onde os índices de pobreza, miséria, desnutrição, mortalidade, analfabetismo, desemprego e infraestrutura socioeconômica estão presentes (IBGE, 2010).

Realmente, as áreas rurais em todo o Brasil possuem piores indicadores de renda, saneamento básico e níveis de escolaridade que as áreas urbanas e, desse modo, acredita-se que isso possa configurar um importante polo de concentração para os agravos da saúde bucal. Por outro lado, sabe-se que a maior concentração de estabelecimentos públicos e privados de saúde está nas zonas urbanas, configurando outro importante fator de desigualdade no acesso aos serviços de saúde. Dessa forma, o reconhecimento das necessidades da população rural é imprescindível para adequar os cuidados de promoção à saúde bucal em relação ao princípio da equidade em saúde, compatibilizando os recursos assistenciais e preventivos às efetivas necessidades da população (MELLO; ANTUNES, 2004; FURTADO;
TRAEBERT; MARCENES, 1999).

Desse modo, é importante conhecer alguns desses recursos preventivos relacionados à prevenção da doença cárie, como a fluoretação da água, que é um bem público indispensáveis para a vida e importante para a saúde pública. A fluoretação das águas na estação de tratamento é obrigatória no Brasil com a Lei Federal 6.050 de 1974, sendo que a Portaria 635 de1975 estabeleceu padrões para a operacionalização da medida, incluindo os limites recomendados para a concentração do fluoreto em razão da média das temperaturas máximas (BRASIL, 1976; RIO GRANDE DO SUL,1999; BRASIL, 2011).

Cabe salientar, que o teor de concentração ideal do íon fluoreto na água destinada ao consumo humano é de $0,8 \mathrm{mgF} / 1$ (miligrama de flúor por litro), no Estado do Rio Grande do Sul, e serão considerados dentro do padrão de potabilidade as águas que apresentarem a concentração de íon fluoreto dentro da faixa de 0,6 a 0,9 mg/l (RIO GRANDE DO SUL,1999). Desse modo, para que se obtenha o benefício maior desse íon fluoreto na prevenção da doença cárie, aliado a um baixo risco de fluorose dentária, são necessárias concentrações de 45 a 94 vezes mais altas que 0,01 $\mathrm{mgF} / \mathrm{l}$, de acordo com média das temperaturas máximas anuais do local (SEMINÁRIO VIGILÂNCIA DA FLUORETAÇÃO DE ÁGUAS, 2011).

Segundo Lopez et al., em 2010, a associação de diferentes formas do uso do íon fluoreto, sem contar o seu excesso, tem sido apontada como causa do aumento na incidência de fluorose dentária em crianças e adolescentes, que é conhecida como uma hipomineralização do esmalte e da dentina dos dentes decíduos e permanentes, que ocorre devido ao excesso de ingestão de íons fluoreto durante a formação dos dentes. Em 1997, Narvai destacou que a não agregação desses elementos na água de abastecimento público é juridicamente ilegal, cientificamente insustentável e socialmente injusta. Entretanto, é importante a preservação de requisitos básicos para a utilização desse método, para garantir a sua segurança e a sua efetividade, bem como a continuidade da utilização do íon fluoreto na prevenção da cárie dentária (GUERRA, 2007; MARIMON, 2006; CURY, TABCHOURY, 2003; NARVAI, 2000).

Finalizando, destaca-se que os cursos de formação acadêmica da área da saúde, a partir da aprovação das Diretrizes Curriculares elaboradas pelos Ministérios da Educação e Saúde, passam a ter como objetivo a formação de profissionais comprometidos com a realidade social, enfocando o processo saúdedoença em todas as suas dimensões e manifestações, considerando o cidadão, a família e a comunidade como integrados à realidade epidemiológica e social. As diretrizes indicam que os profissionais tenham uma formação geral, científica, ética, humanista, reflexiva e crítica, propondo mudanças na forma de ensinar (PONTE, 2008). Para desenvolver um programa de educação por meio do trabalho em saúde respondendo 
às demandas do SUS e às diretrizes curriculares, devese propiciar um cenário novo para a formação de profissionais envolvendo a prática de ações interdisciplinares e as atividades de extensão devem estar vinculadas às atividades de ensino e pesquisa do acadêmico.

Diante desse contexto, o objetivo do presente trabalho foi descrever os resultados de uma atividade de extensão interdisciplinar em saúde que avaliou as condições de saúde bucal de estudantes de idade entre 5 a 15 anos, residentes em uma zona rural do estado do Rio Grande do Sul, avaliando a concentração de íons de fluoreto nas águas de abastecimento naturais da região.

\section{Metodologia}

Os pais e/ou responsáveis pelos alunos da Escola Estadual de Ensino Fundamental Rio Grande do Sul assinaram o termo de consentimento livre esclarecido e enviaram no dia da avaliação odontológica da criança, junto com a ficha sobre os dados socioeconômicos da família. Esse projeto foi aprovado pelo Comitê de Ética em Pesquisa da SMS de Porto Alegre, sob o número 669, processo $\mathrm{n}$. 001.032690.11.8.

Trata-se de um estudo transversal observacional, analítico. A amostra foi composta de 58 estudantes, na faixa etária de 5 a 15 anos de idade, regularmente matriculados na Escola Estadual de Ensino Fundamental Rio Grande do Sul, situada no município de General Câmara, Santo Amaro, Rio Grande do Sul. As crianças de cinco anos matriculadas na referida escola seguiram as normas de 2010 do Conselho Nacional de Educação (CNE): para ingressar no Ensino Fundamental, a criança deve completar seis anos até 31 de março do ano vigente (RIO GRANDE DO SUL, 2010).

Os dados foram coletados no mês de fevereiro de 2016, em uma única ida à referida escola para a realização de um mutirão de saúde realizado pela atividade de extensão universitária intitulada "Ação Integradora da Universidade em Educação e Saúde" da Faculdade de Odontologia da Universidade Federal do Rio Grande do Sul. Esse projeto foi desenvolvido baseado nas diretrizes da ação extensionista a partir de parceria da Universidade Federal do Rio Grande do Sul (UFRGS) com a Secretaria Municipal de Saúde de Santo Amaro e $2^{\mathrm{a}}$ Coordenadoria de Saúde do Estado do Rio Grande do Sul, visando desenvolver vivências a partir de ações que incluam o ensino, a pesquisa e a extensão. $\mathrm{O}$ trabalhou envolveu 2 professores coordenadores e 32 acadêmicos dos cursos de medicina, enfermagem, farmácia, ciências biológicas, odontologia, além da comunidade da única escola do município.
O povoado rural de Santo Amaro se localiza a $121 \mathrm{~km}$ da região metropolitana de Porto Alegre. O cenário criado proporcionou a interação dos saberes acadêmico, profissional e popular, com a intenção de formar profissionais comprometidos com a realidade social, a partir da aproximação entre as práticas, troca de conhecimentos e experiências, vivenciando assim a interdisciplinaridade e a indissociabilidade entre extensão, ensino e pesquisa. Todas as atividades (Escovação supervisionada e atividades lúdicas voltadas à saúde bucal, com entrega de kits para higiene bucal), foram previamente explicadas para o diretor da escola e, em seguida, agendadas para serem realizadas em um sábado; ele convocaria todos os seus 58 alunos para participarem.

As condições de saúde bucal foram avaliadas, utilizando-se dos critérios de presença ou ausência de placa visível (Índice de Placa Visível - IPV), sangramento gengival (Índice de Sangramento Gengival - ISG), fluorose (Índice de Dean) e número de dentes cariados, perdidos e obturados (Índices ceod e CPOD). Esses exames bucais foram realizados por cinco acadêmicos de odontologia do último semestre, capacitados para realizar os referidos exames, mediante luz artificial nos ambientes mais claros da escola, sendo iniciados pela verificação da presença de placa visível, seguida da escovação supervisionada e a observação de pontos de sangramento gengival. Após essa deplacagem com escova dentária e a secagem dos dentes com uma gaze, sob isolamento relativo, levantou-se o número de dentes cariados, perdidos, obturados, presença e ausência de fluorose (Indice de Dean). (Fig. 1)

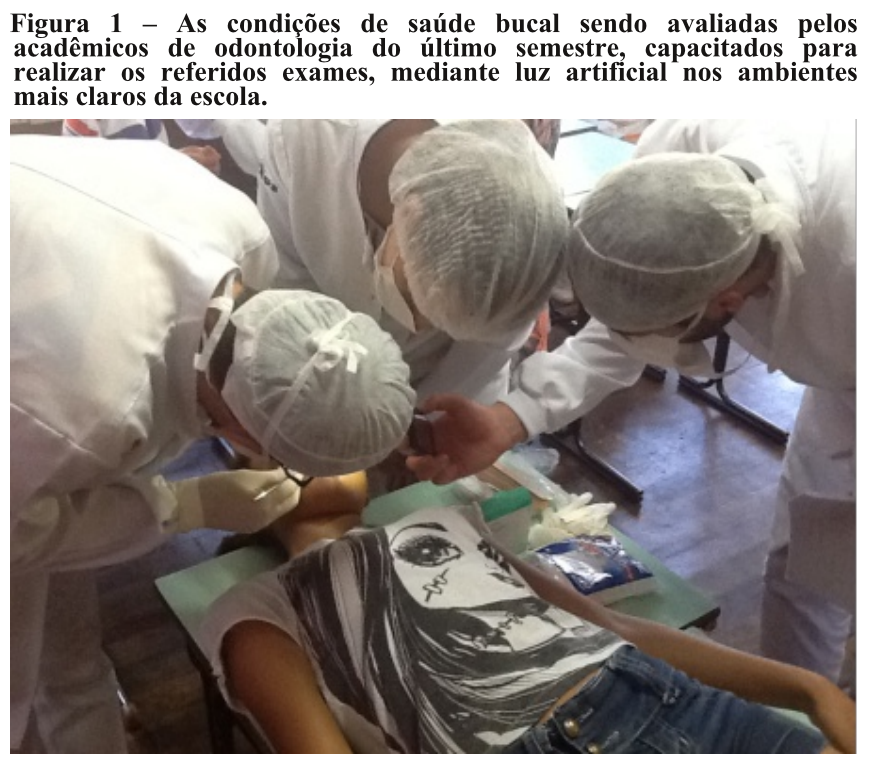

Adicionalmente, como sendo uma região rural, sem saneamento básico na maioria das casas, a água provém de poços artesianos. Desse modo, foram analisadas as águas quanto à concentração de fluoreto, coletando amostras distintas do distrito de Santo Amaro para o consumo da população local. Essas amostras foram adquiridas dos três poços artesianos abertos que abastecem a comunidade (poço A, B e C), durante a visita à Escola de Ensino Fundamental Rio 
Grande do Sul; havia mais dois poços artesianos que eles interditaram ao saber de nossa visita à região.

Inicialmente, as águas foram armazenadas em condições de temperatura e rotuladas com seguintes informações: tipo de água (poço artesiano) e local de origem. Posteriormente, foram acondicionadas em uma caixa de isopor e transportadas para análise. Todos os frascos foram numerados de forma a possibilitar a identificação dos seus conteúdos posteriormente, sem, no entanto, interferir na identificação no momento da análise.

As amostras foram analisadas em duplicata, através de um eletrodo específico para flúor (Orion 9609, Orion Research Inc., Beverly, Massachusetts, USA) conectado a um analisador de íons (Procyon, São Paulo, Brazil). Calibrações em duplicata foram realizadas previamente com soluções padrão de íon fluoreto contendo de 0,1 a $1,0 \mu \mathrm{g} \mathrm{F} / \mathrm{mL}$, preparados em TISAB II (Total Ionic Strength Adjustment Buffer) a $50 \%$. Foi utilizado o método de leitura direta, após a colocação de $1 \mathrm{~mL}$ da amostra de água e $1 \mathrm{~mL}$ de TISAB II. Todas as leituras (de calibração e das amostras) foram obtidas em $\mathrm{mV}$ (milivolts) e transformadas por meio de uma curva de calibração de regressão linear em concentrações de íon fluoreto $\mathrm{mgF} / \mathrm{L}$, com o auxílio de uma planilha de cálculos através do programa Microsoft Excel 2013.

Os resultados, com relação à saúde bucal e nível socioeconômico, foram compilados em um banco de dados no programa Microsoft Excel 2013, para serem analisados e expressos em porcentagem.

\section{Resultados}

Dos 58 escolares examinados, três foram excluídos por não preencherem os questionários adequadamente, finalizando a amostra em 55 escolares, sendo 23 do sexo feminino $(41,81 \%)$ e 32 do sexo masculino $(58,18 \%)$. A idade das crianças variou entre 5 e 12 anos de idade, sendo 9 anos a média obtida. A maioria dos examinados não fazia uso continuo de medicação $(89,09 \%$ - $\mathrm{n}=49)$ e praticavam atividade física semanalmente $(69,10 \%-\mathrm{n}=35)$.

Com relação aos indicadores socioeconômicos, que foram respondidos pelos responsáveis dos alunos, $67 \%(n=37)$ dos cuidadores possuíam apenas o $1^{\circ}$ grau incompleto de escolaridade e $3,63 \% \quad(n=2)$ eram analfabetos; a maioria vivia com renda mensal de até um salário mínimo nacional $(83,63 \%-\mathrm{n}=46)$, conforme tabela 1.

Em relação ao tipo de moradia dos examinados, $40 \%(\mathrm{n}=22)$ residiam em casa de alvenaria, $34,55 \%$ $(n=19)$ em casa de madeira e $24,45 \%(n=14)$ em residência mista; $70,90 \%(\mathrm{n}=39)$ das famílias eram compostas de 3 a 5 pessoas (Tabela 2). A maioria dos participantes possuía banheiro no corpo da casa, mais de três torneiras nas residências e seus dejetos eram depositados em fossas, $90,9 \%(n=50), 81,3 \%(n=45)$ e $70.9 \%(\mathrm{n}=39)$, respectivamente.

\begin{tabular}{c|c|c|c|c|}
$\begin{array}{l}\text { Tabela } 1 \text { - Renda Familiar e Escolaridade - Renda Familiar Total em } \\
\text { Porcentagem de Salários Mínimos por Família e porcentagem de } \\
\text { Escolaridade dos Chefes de Família }\end{array}$ & $\begin{array}{c}\text { por } \\
\text { Renda Familiar Total }\end{array}$ & $\%(n)$ & Escolaridade & $\%(n)$ \\
\hline Menos de 1 SM & $83,63(n=46)$ & $1^{\circ}$ grau incompleto & $67,2(n=37)$ \\
\hline 1 a 2 SM & $16,36(n=9)$ & $1^{\circ}$ grau completo & $12,72(n=16)$ \\
\hline 3 a 5 SM & $0(n=0)$ & Analfabeto & $3,6(n=2)$ \\
\hline
\end{tabular}

\begin{tabular}{|c|c|c|c|}
\hline & Até 2 & $\begin{array}{c}\%(n) \\
\operatorname{De} 3 \text { a } 5\end{array}$ & Mais de 5 \\
\hline $\begin{array}{l}N^{\circ} \text { de Moradores por } \\
\text { domicilio }\end{array}$ & $5,45 \%(n=3)$ & $70,9 \%(n=39)$ & $23.65 \%(n=13)$ \\
\hline \multirow[t]{2}{*}{$\mathrm{N}^{\circ}$ de Peças no Domicilio } & $10,9 \%(n=7)$ & $56.36 \%(n=30)$ & $32,74 \%(n=18)$ \\
\hline & Alvenaria & $\begin{array}{c}\%(n) \\
\text { Madeira }\end{array}$ & Mista \\
\hline Tipo de Moradia & $40,0 \%(n=22)$ & $34,55 \%(n=19)$ & $25,45 \%(n=14)$ \\
\hline
\end{tabular}

Tabela 3 - Ingestão de açúcar e Higiene bucal - Porcentagem de pessoas conforme a frequência de ingestão de açúcar e higiene bucal por dia.

\begin{tabular}{|c|c|c|}
\hline & $\begin{array}{c}\text { Frequência de Ingestão de } \\
\text { Açúcar \% (n) }\end{array}$ & $\begin{array}{c}\text { Frequência de } \\
\text { por dia } \%(n)\end{array}$ \\
\hline 1 vez ao dia & $18,18 \%(n=10)$ & $3,63 \%(n=2)$ \\
\hline 2 a 3 vezes ao dia & $49,09 \%(n=26)$ & $89,09 \%(n=49)$ \\
\hline Mais de 3 vezes ao dia & $32,73 \%(n=18)$ & $7,28 \%(n=4)$ \\
\hline
\end{tabular}

Na verificação de saúde bucal, a prevalência de cárie dentária foi de $45,50 \%(\mathrm{n}=25) ; 5$ anos - 3,62\% $(\mathrm{n}=2) ; 6$ anos $-3,62 \%(\mathrm{n}=2) ; 7$ anos $-5,46 \%(\mathrm{n}=3) ; 8$ anos $-3,62 \%(\mathrm{n}=2) ; 9$ anos $-1,82 \%(\mathrm{n}=1) ; 10$ anos $5,46 \%(\mathrm{n}=3) ; 11$ anos - $1,82 \%(\mathrm{n}=1) ; 12$ anos $7,28 \%(\mathrm{n}=4) ; 13$ anos $-5,46 \%(\mathrm{n}=3) ; 14$ anos $-3,62 \%$ $(\mathrm{n}=2) ; 15$ anos - 3,62\% (n=2). 56\% (n=31) dos participantes apresentaram placa evidente, enquanto $44 \%$ não possuíam placa visível ao exame clinico. Quanto à presença de sangramento gengival, 67\% $(n=37)$ não apresentaram se quer um sitio sangrante, enquanto $33 \%$ apresentaram sangramento devido à escovação supervisionada (Figura 2). 
Figura 2 - Porcentagem de Presença de Placa Visível (IPV) e Sangramento Gengival (ISG) por gênero

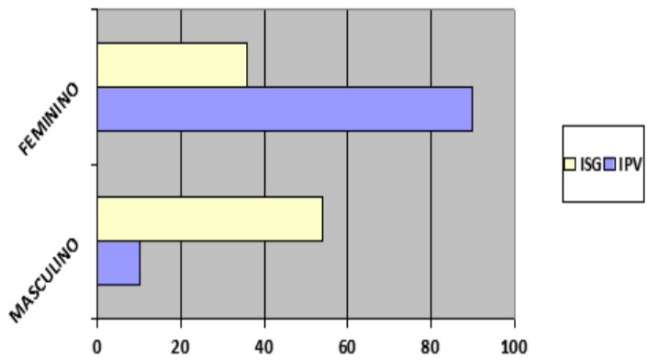

Quanto ao questionário odontológico, 74,5\% $(n=41)$ os escolares afirmaram que em algum momento receberam alguma instrução sobre higiene bucal e que teria sido na própria escola. Desses, $40 \%(n=22)$, que eram as crianças de 7 a 15 anos de idade, não receberam supervisão em casa para a sua escovação dentária e $60 \%(n=32)$ haviam sido instruídos, por serem menores de idade e terem seus pais recebido algum tipo de orientação quanto à higiene bucal na própria escola.

Completando o exame dentário, 94\% $(\mathrm{n}=52)$ dos alunos não possuíam restaurações e $6 \% \quad(n=3)$ apresentaram algum elemento dentário restaurado. $52 \%(n=29)$ possuíam lesões de cárie e $48 \%(n=26)$ não apresentavam. Em relação às perdas dentárias, a totalidade $(\mathrm{n}=55)$ dos examinados não apresentaram perdas dentárias e 14,54\% $(n=8)$ tinham dentes com extração indicada por se tratarem de restos radiculares; $85,45 \%(n=47)$ não necessitam de exodontias.

Um dado que chamou atenção durante a coleta de dados sobre a saúde bucal dos alunos foi a alta incidência de fluorose, uma vez que, do total de 55 crianças, $52,72 \% \quad(\mathrm{n}=29)$ apresentavam a variável fluorose de maneira moderada a severa, segundo o índice de Dean. A maior incidência de fluorose estava nos alunos do sexo masculino, totalizando 19 (Figuras 3 e 4$)$.

Figura 3 - Fluorose presente nos escolares - Segundo Índice de Dean fluorose moderada: Toda a superfície afetada, superfícies sujeitas ao desgaste, manchas amarronzadas frequentes.

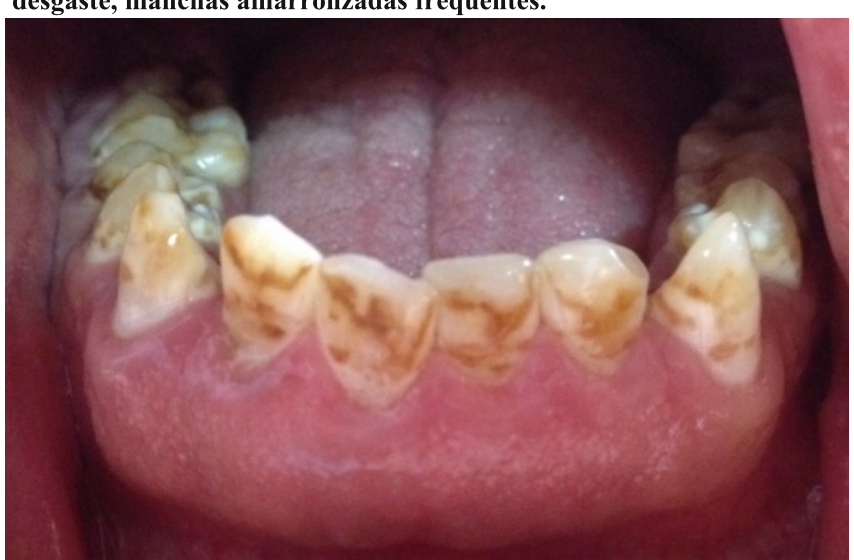

Figura 4 - Fluorose presente nos escolares - Segundo Índice de Dean fluorose severa: Toda a superfície afetada, mudança na anatomia, manchas marrons, erosões e aparência de corrosão.

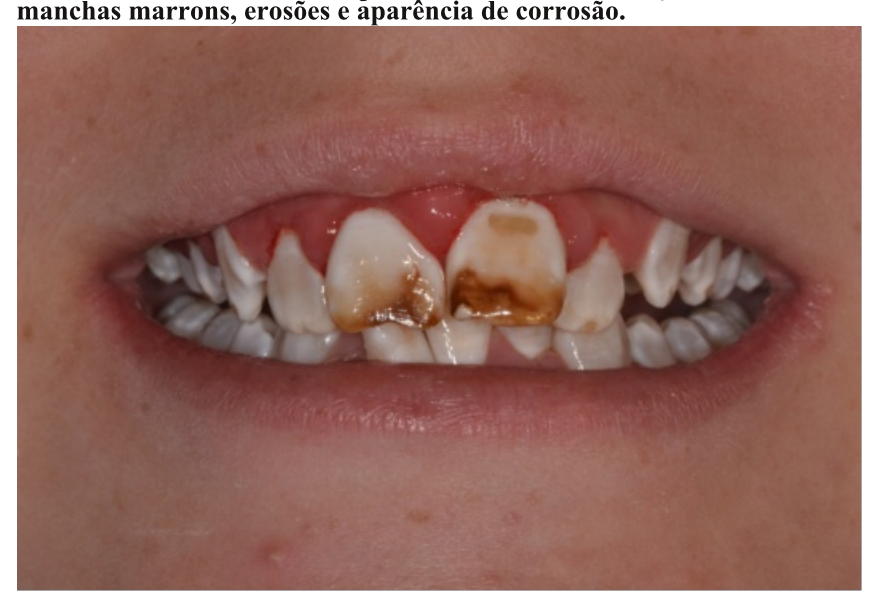

Quanto à concentração de íons fluoreto encontrada nas amostras coletadas nos três poços artesianos A, B e C, que hoje abastecem a comunidade de Santo Amaro, foram de 1,18, 1,19 e 1,29 ppm, respectivamente.

\section{Discussão}

Contextualizar a saúde no campo é muito importante atualmente, pois esse tema é pouco discutido no contexto geral (MACHADO; KNORST, 2010). A população pesquisada foi de estudantes de uma comunidade rural e, embora as matrículas escolares no campo representem apenas $13 \%$ do total do Brasil, esse percentual representa mais de 6,6 milhões de crianças e jovens espalhados em 83 mil escolas rurais brasileiras (FERNANDES, 2011).

Segundo Machado e Knorst (2010), o tema saúde no campo é pouco discutido em seu aspecto social, uma vez que as escolas rurais vivem uma dinâmica diferente de sistema educacional, ou pelo menos deveriam viver. Para Figueiredo et al. (2014a), as famílias dos alunos da Escola Rural de Ensino Fundamental Rio Grande do Sul sobreviviam com menos de um salário mínimo nacional por mês e, em sua maioria, eram compostas por 3 a 5 moradores por residência, em situação precária. Isso reflete a realidade vivida também por 16,2 milhões de pessoas consideradas extremamente pobres, o equivalente a $8,5 \%$ da população. Os dados do instituto apontam que os 16,267 milhões de brasileiros extremamente pobres estão concentrados principalmente na região Nordeste, totalizando 9,61 milhões de pessoas (59,1\%), sendo a maioria no campo - 56,4\% (AGENCIA BRASIL, 2011).

Essa realidade foi encontrada na comunidade analisada, demonstrando uma relação proporcional entre renda dos moradores e a presença das doenças bucais mais prevalentes: cárie e periodontal. Esses dados corroboraram com resultados das pesquisas de 
Figueiredo et al., em 2011 e 2014a, que também concluíram haver associação entre a ocorrência de cárie dentária e presença de gengivite com o nível socioeconômico de uma população de baixa renda.

É interessante ressaltar também que a maior parte das famílias neste estudo relatou viver em péssimas condições de moradia; apesar de grande parte ser de casas de alvenaria, estas eram de 3 a 5 cômodos, abastecidas com água proveniente de poços artesianos e sem saneamento básico. Agregando a essa falta de condições adequadas de infraestrutura, o alto número de moradores por domicílio potencializou os problemas encontrados.

Desse modo, neste estudo, a cárie dentária pôde ser considerada como um forte indicador de desigualdade social, uma vez que foi encontrada em $45,5 \%$ dos escolares avaliados. Desde o Projeto SBBrasil de 2003 do Ministério da Saúde, já se observava uma desigualdade: cerca de $14 \%$ dos adolescentes brasileiros nunca havia ido ainda ao dentista, sendo um dos principais motivos da ida ao dentista a experiência de dor dentária, a qual foi relatada por mais de $30 \%$ participantes; a consulta de rotina/manutenção foi relatada por cerca de $30 \%$ dos adolescentes (BRASIL, 2004).

Ao avaliar os resultados clínicos deste estudo, pode-se também perceber que os escolares apresentaram alto Índice de Placa Visível (IPV) e Sangramento Gengival (ISG), refletindo baixo grau de conhecimento e de motivação para o autocuidado com a saúde bucal, uma vez que se sabe da importância da instrução de higiene bucal ser acompanhada de motivação e reforços periódicos para a redução do IPV e ISG (TOASSI; PETRY, 2002). Na amostra aqui avaliada, quase $90 \%$ dos escolares disseram realizar sua higiene bucal 3 vezes ao dia, dado este que não coincidiu com os achados clínicos deste estudo, evidenciando claramente que a afirmativa não condiz com a realidade do seu dia a dia ou que o grau de conhecimento dos estudantes a respeito de técnica de higiene bucal foi insuficiente.

Com relação à ingestão de açúcar, neste estudo, $50 \%$ das famílias consumiam açúcar três vezes ao dia. Esse resultado é semelhante ao de Figueiredo et al., em 2014b, ao descreverem sobre as condições de saúde bucal e o padrão alimentar de crianças também residentes em um bairro pobre de uma cidade do sul do Brasil, verificando que $64,6 \%$ dos cuidadores também escolheram a opção " 3 vezes ao dia" para o consumo de açúcar.

Um agravante a mais encontrado neste estudo foi que $52,7 \%$ dos escolares examinados apresentaram fluorose dentária, que é um distúrbio de desenvolvimento que afeta o esmalte durante sua formação. Essa alteração resulta de uma injúria contínua, de baixa intensidade e longa duração na fase de maturação do esmalte dentário (SUCKLING, 1989), geralmente sendo provocada pela ingestão de múltiplas fontes de fluoreto (CURY; TABCHOURY, 2003). Assim sendo, em algum momento do desenvolvimento dentário dessas crianças, elas foram submetidas a concentrações inadequadas de íons fluoreto, ou seja, um pouco maior que a ideal, provavelmente oriundos da água de abastecimento público da região, que é proveniente de poços artesianos. Dessa maneira, crê-se que a fluorose moderada e severa (ou grave) encontrada, segundo o índice Dean, não foi apenas afetada pelos teores de íons fluoreto encontrado nos poços $\mathrm{A}, \mathrm{B}$ e C avaliados, mas também, possivelmente, quando eles consumiram a água dos dois poços artesianos que foram lacrados pelos gestores do município pouco tempo antes de nossa chegada na comunidade.

A incidência de fluorose encontrada nessa população está acima da média encontrada na literatura. Um estudo epidemiológico desenvolvido no estado de São Paulo apontou um percentual de fluorose que variou entre $16,8 \%$ e $34,5 \%$ em distintas cidades (GUERRA, 2017); Rigo et al., em 2010, avaliaram estudantes de escolas de Passo Fundo, no Rio Grande do Sul, e encontraram $32,8 \%$ de prevalência de fluorose, mas o grau de severidade foi baixo; ainda no estado do Rio Grande do Sul, outros trabalhos demonstraram preocupação com o excesso de íons de fluoreto nas águas de abastecimento naturais, como no município de Santa Teresa, onde foi encontrada uma prevalência de $63,7 \%$ de fluorose e, mais uma vez, o grau de severidade muito leve foi o predominante $(43,6 \%)$. É importante ressaltar que, nesse último estudo, também foram avaliadas outras fontes de fluoreto, as quais essas crianças foram expostas (TOASSI; ABBEG, 2005).

Finalizando, deve-se reconhecer a necessidade de uma maior precisão e fortalecimento das medidas de vigilância sanitária na área rural do município de General Câmera, incluindo uma melhor ação da vigilância em Saúde (SVS/MS) na qualidade da água para consumo humano, em articulação com as Secretaria de Saúde do Estado do Rio Grande do Sul (BRASIL, 2011). Para tanto, são importantes que sejam realizadas atividades extensionistas, interdisciplinares e intersetoriais em regiões como a avaliada neste estudo, visando à formação e capacitação de acadêmicos comprometidos com a realidade social e regional, conforme o perfil solicitado pelo estado do Rio Grande do Sul.

\section{Conclusões}

Após analisar o perfil da saúde bucal dos escolares da Escola Estadual de Ensino Fundamental Rio Grande do Sul, no distrito de Santo Amaro, Rio Grande do Sul, conclui-se que a população de 
escolares de 5 a 15 anos de idade apresentou altos índices de placa visível, sangramento gengival, fluorose, dentes cariados, perdidos e obturados. Os teores de íons de fluoreto nas águas de abastecimento natural não se encontravam dentro dos padrões de normalidade.

\section{Agradecimentos}

Agradecemos ao diretor da Escola Estadual Ensino Fundamental Rio Grande do Sul - Sr. André -, professores envolvidos no dia do mutirão de saúde e toda a comunidade de Santo Amaro pela colaboração na execução deste trabalho.

\section{Referências}

AGÊNCIA BRASIL. Laboratório de Demografia e Estudos Populacionais. Cerca de 16,2 milhões de brasileiros são extremamente pobres: a maioria é jovem, negra e nordestina. Brasil, 2011. Disponível em: $<$ http://www.ufff.br/ladem/2011/05/05/cerca-de-162milhoes-de-brasileiros-sao-extremamente-pobres-a-maioriae-jovem-negra-e-nordestina/>. Acesso em: 21 jun. 2017.

BALDANI, M. H.; VASCONCELOS, G. A.; FERREIRA, L. J. Associação do índice CPO-D com indicadores socioeconômicos e de provisão de servicos odontológicos no Estado do Paraná, Brasil. Cad. Saúde Pública, Rio de Janeiro, v. 20, p. 143-52, mai. 2004.

BRASIL. Ministério da Saúde. Portaria n. 635, de 26 de dezembro de 1975. Diário Oficial [da] República Federativa do Brasil, Brasília, 30 jan. 1976. Disponível em:

$<$ http://lproweb.procempa.com.br/pmpa/prefpoa/sms/usu do c/portaria635.pdf>. Acesso em: 6 mai. 2017.

BRASIL. Ministério da Saúde. Portaria $n^{\circ} .2 .914$, de 12 de dezembro de 2011. Diário Oficial [da] República

Federativa do Brasil, Brasília, 14 dez. 2011. Seção 1, p. 39. Disponível em:

$<$ http://site.sabesp.com.br/uploads/file/asabesp doctos/kit_a rsesp portaria2914.pdf $>$. Acesso em: 6 mai. 2017.

BRASIL. Ministério da Saúde. Secretaria de Atenção à Saúde. Secretaria de Vigilância em Saúde. Departamento de Atenção Básica. Coordenação Geral de Saúde Bucal. Projeto SBBrasil 2003. Brasília, 2004. Disponível em: $<$ http://cfo.org.br/wp-

content/uploads/2009/10/04_0347_M.pdf>. Acesso em: 6 jun. 2017.

BRASIL. Ministério da Saúde. Secretaria de Atenção à Saúde. Secretaria de Vigilância em Saúde. Departamento de Atenção Básica. Coordenação Geral de Saúde Bucal.

Projeto SBBrasil 2010. Disponível em:

$<$ http://dab.saude.gov.br/CNSB/sbbrasil/arquivos/projeto sb 2010_relatorio_final.pdf>. Acesso em: 26 maio 2017.

BRASIL. Ministério do Desenvolvimento Social e Combate à Fome. Brasil sem miséria atenderá 16,2 milhões. Portal Brasil, Brasília, 3 mai. 2011. Disponível em:

$<$ http://www.brasil.gov.br/governo/2011/05/brasil-semmiseria-atendera-16-2-milhoes>. Acesso em: 6 jun. 2017.
CAMARGO, M. G. A.; NATERA, A. Nivel de conocimiento de defectos de esmalte y su tratamiento entre odontopediatras. Rev. Odontopediatr. Latinoam., v. 7, no. 1, p. 25-35, jan./jun. 2017. Artículo 4. Disponível em: $<\mathrm{http}$ ///www.revistaodontopediatria.org/ediciones/2017/1/art $-4 />$. Acesso em: 6 jun. 2017.

CAMPOS, L. et al. Conhecimento de mães de diferentes classes sociais sobre saúde bucal no município de Cocal do Sul (SC). Rev. Sul-Bras. Odontol., Joinville, v. 7, n. 3, p. 2872-2895, ago. 2010.

CURY, A. J.; TABCHOURY, C. P. Determination of appropriate exposure to fluoride in non-EME countries in the future. J. Appl. Oral Sci., Bauru, v. 11, n. 2, p. 83-95, jun. 2003.

FERNANDES, E. Desigualdades em campo. Rev. Educ., São Paulo, 10 set. 2011. Disponível em: $<$ http://www.revistaeducacao.com.br/desigualdades-emcampo/>. Acesso em: 6 jun. 2017.

FIGUEIREDO, M. C. et al. Descrição do padrão alimentar e da saúde bucal de crianças do sul do Brasil. RFO, Passo Fundo, v. 19, n. 3, p. 329-336, set./dez. 2014b.

FIGUEIREDO, M. C. et al. Saúde bucal de moradores de um bairro pobre de Xangri-Lá, RS, Brasil. ConScientiae Saúde, São Paulo, v. 10, n. 2, p. 292-298, jun. 2011.

FIGUEIREDO, M. C. et al. Saúde bucal de pessoas em situação de pobreza extrema residentes em um município no Sul do Brasil. UNOPAR Cient. Ciênc. Biol. Saúde, v. 16, p. $45-50$, dez. $2014 \mathrm{a}$.

FURTADO, A.; TRAEBERT, J. L.; MARCENES, W. S. Prevalência de doenças bucais e necessidade de tratamento em Capão Alto, Santa Catarina. Rev. ABO Nac., Rio de Janeiro, v. 7, p. 226-230, jul. 1999.

GAMBHIR, R. S. et al. Impact of school based oral health education programmes in India: a systematic review. J. Clin. Diag. Res., Delhi, v. 7, no. 12, p. 3107-3110, dez. 2013.

GUERRA, L. M. Cárie e fluorose: relação com variáveis socioeconômicas e demográficas em municípios com diferentes concentrações de flúor nas águas de abastecimento público. 2007. 84 f. Dissertação (Mestrado), Faculdade de Odontologia de Piracicaba, Universidade Estadual de Campinas, Piracicaba, 2007.

IBGE. Instituto Brasileiro de Geografia e Estatística. Sinopse do censo demográfico 2010 Brasil. Brasília, 2010. Disponível em:

$<$ http://www.censo2010.ibge.gov.br/sinopse/index.php?dado $\mathrm{s}=11 \& u f=00>$. Acesso em: 8 jun. 2017.

KUMAR, J. V. et al. Dean's fluorosis index: an assessment of examiner reliability. J. Public Health Dent., Raileigh, v. 60 , no. 1, p. $57-59,2000$

LOPEZ, I. Y. et al. Dental caries in preschoolers from communes with fluoridated and non-fluoridated public water supplies in Chile. Rev. Odonto Ciência., v. 25, p. 20-24, fev. 2010 .

MACHADO, D. L.; KNORST, P. A. R. A gestão democrática presente na escola do campo: o caso da Escola de Ensino Fundamental Linha Biguá - SC. Unoesc \& Ciência - ACHS, Joaçaba, v. 1, n. 2, p. 111-120, jul./dez. 2010.

MARIMON, M. P. C. O flúor nas águas subterrâneas da formação Santa Maria, na regia de Santa Cruz e Venâncio Aires, RS, Brasil. 2006. 246 f. Tese (Doutorado), Instituto de Geociências, Universidade Federal do Rio Grande do Sul, Porto Alegre, 2006.

MELLO, T. R. C.; ANTUNES, J. L. F. Prevalência de cárie dentária em escolares da região rural de Itapetininga, São Paulo, Brasil. Cad. Saúde Pública, Rio de Janeiro, v. 20, n. 3, p. 829-835, jul. 2004. 
NARVAI, P. C. Fluoretacão das águas: razões para prosseguir. São Paulo: FUNDAP/SES-SP, 1997.

PONTE, C. I. R. V. Identificação e discussão do processo de produção/construção do conhecimento a partir das acões de extensão realizadas pelos professores da

FAMED/UFRGS no período 2000-2004. 2008. $100 \mathrm{f}$. Tese (Doutorado), Programa de Pós-Graduação em Cardiologia e Ciências Cardiovasculares, Faculdade de Medicina, Universidade Federal do Rio Grande do Sul, Porto Alegre, 2008.

RIGO, L. et al. Estudo sobre a fluorose dentária num município do Sul do Brasil. Ciênc. Saúde Colet., Rio de Janeiro, v. 15, p. 1439-1448, 2010.

RIO GRANDE DO SUL. Conselho Estadual de Educação (CEED). Resolução CEED, $\mathbf{n}^{\circ}$ 307, de 31 de março de 2010. Porto Alegre, 2010. Disponível em: $<$ http://www.ceed.rs.gov.br/conteudo/9379/qual-a-idademinima-exigida-para-ingressar-no- $1 \% \mathrm{C} 2 \% \mathrm{~B} 0$-ano-doensino-fundamental $\% 3 \mathrm{~F}$-qual-a-idade-de-corte $\% 3 \mathrm{~F}>$. Acesso em: 6 jun. 2017.

RIO GRANDE DO SUL. Secretaria Estadual da Saúde. Coordenadoria de Atenção Integral à Saúde. Divisão de Vigilância Sanitária. Portaria n. ${ }^{\circ}$ 10/99, 1999. Porto Alegre, 1999. Disponível em:

$<$ http://lproweb.procempa.com.br/pmpa/prefpoa/sms/usu_do c/portaria_10 99.pdf>. Acesso em: 6 mai. 2017.
SEMINÁRIO VIGILÂNCIA DA FLUORETAÇÃO DE ÂGUAS (2011, São Paulo). Consenso técnico sobre classificação de águas de abastecimento público segundo o teor de flúor. São Paulo: USP. Faculdade de Saúde Pública. Centro Colaborador do Ministério da Saúde em Vigilância da Saúde Bucal (CECOL), 2011. Disponível em: $<$ http://www.cecol.fsp.usp.br/dcms/uploads/arquivos/13981 77715 CECOL-USP-

ClassificacaoAguasSegundoTeorFluor-

DocumentoConsensoTecnico-2011.pdf $>$. Acesso em: 28 jun. 2017.

SPOLIDORIO, D. M. P. et al. Dental caries status in decíduos and permanent dentition of Brazilian children aged 6-8 years with a socio-economic base. Braz. J. Oral Sci., Piracicaba, v. 2, p. 147-150, 2003.

SUCKLING, G. W. Developmental defects of enamel historical and present-day perspectives of their pathogenesis. Adv. Dent. Res., v. 3, n. 2, p. 87-94, 1989.

TOASSI, R. F. C.; ABBEG, C. Fluorose dentária em escolares de um município da serra gaúcha, Rio Grande do Sul, Brasil. Cad. Saúde Pública, Rio de Janeiro, v. 21, n. 2, p. 652-655, abr. 2005.

TOASSI, R. F. C.; PETRY, P. C. Motivação no controle do biofilme dental e sangramento gengival em escolares. Rev. Saúde Pública, São Paulo, v. 36, n. 5, out. 2002. 